\title{
Stimuler la coopération organisationnelle en regard de la diversité internationale des travailleurs : proposition d'un outil de gestion fondé sur la lieuité
}

\author{
Carène Tchuinou ${ }^{a}$, Anne-Laure Saives ${ }^{b}$
}

RÉSUMÉ. Cet article vise à comprendre la coopération entre travailleurs immigrants à partir d'une analyse de leurs perceptions du lieu d'implantation de leur organisation. Partant des expériences de quelques travailleurs camerounais de Montréal, nous avons découvert ce lieu comme une construction aux multiples facettes (géographique, locale et symbolique). Une fois la grille d'analyse « lieuitaire » établie, nous avons entrepris une activité de promenade commentée qui, à partir d'un itinéraire proposé par les participants, nous a permis d'identifier des éléments garants d'une meilleure coopération organisationnelle. Par un intérêt porté au partage des sens du lieu individuels, nous avons découvert que la réalisation d'une telle activité au sein des équipes de travail peut être porteuse d'une plus grande humanisation du travail et permettrait de mieux faire valoir la complémentarité des expériences et des savoirs individuels, des notions clés pour le partage, la création de connaissances, la collaboration et la coopération organisationnelle réussies.

\begin{abstract}
This paper aims to understand the organizational cooperation between immigrant workers, based on an analysis of their perceptions of the place. Based on the experiences of some Cameroonian workers in Montreal, we discovered this place as a multi-faceted construction (geographical, local and symbolic). With a "place-based" analysis grid, we initiated a commented walk activity inspired by an itinerary proposed by the participants. This activity allowed us to identify relevant elements that would guarantee better organizational cooperation. Through an interest in the sharing of the individual senses of the place, we discovered that such activity within work teams could be source of a greater bumanization at work. It would make it possible to better emphasize the complementarity of experiences and individual knowledge, key concepts for knowledge sharing, knowledge creation, collaboration and successful organizational cooperation.
\end{abstract}

\section{Introduction}

Le profil professionnel des individus qui composent désormais l'effectif des entreprises n'est plus homogène. La globalisation et la mondialisation des économies et des entreprises ont eu un impact fort sur le management des organisations et les modes de gestion des ressources humaines. En effet, les entreprises emploient des salariés de diverses origines, en provenance de divers pays et donc, de cultures différentes. De plus, la mobilité des entreprises à l'échelle internationale suppose une gestion de ressources humaines désormais réparties dans plusieurs pays. Ces réalités imposent ainsi aux organisations et aux managers la nécessité de faire collaborer et coopérer au sein d'une même organisation des individus aux différences multiples. Si plusieurs auteurs nous ont précédés dans une tentative de définition d'une gestion organisationnelle tenant mieux compte de l'hétérogénéité des individus au sein des organisations (P. Dupriez, S. Simons, E. Mutabazi, P. Pierre, O. Meier, D. Loth, etc.), nous envisageons ici de poursuivre sur cette lancée en utilisant une approche fondée sur un concept peu abordé en management, celui de lieu. Nous pensons en effet que la dimension «lieuitaire » participe de façon fondamentale à la compréhension des actions des individus au sein

\footnotetext{
a Candidate au doctorat (Ph. D.) en sciences de l'administration (Management), Université Laval

b Professeure, Département de management et technologie, ESG-UQAM
} 
des organisations. En effet, à notre sens, les perceptions de nouveaux arrivants au sein d'un lieu d'accueil influencent inéluctablement leur travail et leur rendement. Les différences de perceptions du lieu par les individus au sein des organisations, et surtout leur partage, finissent par créer une nouvelle forme de coopération plus authentique, plus durable et moins superficielle. Il devient ainsi urgent de s'interroger sur la place réservée aux perceptions lieuitaires des nouveaux occupants d'un lieu géographique, et d'observer leur impact sur la transformation de leur cadre de vie, leur espace habité et surtout leurs relations de travail. Précisément, comment la mobilisation du concept de lieu participe-t-elle à la transformation des liens de coopération et à la redéfinition du management au sein d'une organisation à la main-d'œuvre culturellement diverse?

La réponse à cette question passe d'abord par une brève définition de la notion de lieu, ce qui nous permettra par la suite d'identifier ses influences sur la coopération organisationnelle pour être en mesure d'opérer les choix stratégiques qui s’imposent.

Ainsi, dans cette étude, nous avons approché la réalité de la coopération entre travailleurs d'origines diverses à partir du vécu de travailleurs immigrants camerounais dans de grandes entreprises au Québec. Pour saisir les raisons ou les freins à la collaboration interindividuelle en contexte internationalisé, nous proposons un outil de collecte de données original, la «Promènade », dont nous décrirons la nature, l'utilité et les limites dans cet article.

Toutefois, il importe au préalable de situer le contexte et le cadre général de notre étude, par la présentation de quelques données sur l'immigration et la population immigrante au Canada, ainsi que les transformations du monde de l'entreprise à la suite de cette vague d'immigration.

\section{Quelques données sur l'immigration et les transformations de l'entreprise}

Durant la période 2015-2016, le Canada a accueilli un peu plus de 320000 immigrants, un nombre record pour le pays qui en reçoit en moyenne 250000 chaque année ${ }^{1}$. Selon les nouvelles données de Statistique Canada, 320932 personnes ont immigré au Canada du $1^{\text {er }}$ juillet 2015 au 30 juin 2016. Les quelque 31000 réfugiés syriens qui ont été admis au Canada depuis novembre 2015 ont contribué à ce chiffre record. Cette immigration massive vise essentiellement à prévenir le vieillissement de la population active et la pénurie de la main-d'œuvre. Le Canada adopte en particulier une politique d'immigration choisie, dans laquelle la plupart des personnes sélectionnées montrent un profil jeune et un niveau d'instruction relativement élevé par rapport aux années précédentes ${ }^{2}$. Selon les résultats de la dernière Enquête nationale auprès des ménages (ENM) publiée par Statistique Canada en 2013, un Canadien sur cinq $(20,6 \%)$ est né à l'étranger, soit 6775800 personnes $^{3}$. Dans le cas particulier du Québec, le flux d'entrée des immigrants légaux au Québec est actuellement de 51500 par an ${ }^{4}$. La province en particulier continue d'attirer les immigrants; en effet, près d'un nouvel arrivant sur cinq s'établit au Québec et en particulier dans la région métropolitaine de Montréal. Cette dernière reste la deuxième ville au pays à accueillir le plus d'immigrants, derrière Toronto. En 2011, la métropole comptait à elle seule 846645 immigrants, soit $12,5 \%$ du total national. De plus, la ville constitue un attrait incontournable, loin devant les autres municipalités du Québec ${ }^{5}$. La région de Montréal reste ainsi la destination la plus populaire et continue de regrouper la grande majorité des immigrants avec $71 \%$ qui projetaient s'y installer en 2013. Ces chiffres justifient à eux seuls notre intérêt à nous interroger sur les nouveaux enjeux du management des entreprises devant cette vague d'immigration.

En effet, devant ce phénomène qui ne cesse de gagner en ampleur, les dirigeants et les managers doivent faire face à certains défis; ces nouvelles dynamiques les forcent à revoir les modèles de gestion à partir desquels ils dirigent l'entreprise et structurent leur environnement de travail ${ }^{6}$. Ils doivent composer avec: une diversité cognitive des employés par la mise en relation d'individus d'expériences et d'origines différentes, nécessitant ainsi de nouvelles capacités à communiquer; une globalisation des talents qui suppose de nouvelles mesures de recrutement, mais aussi d'incitation et de persuasion des individus; et une diversité même des activités de l'entreprise qui est désormais contrainte à mettre sur pied de nouvelles mesures destinées à favoriser l'intégration et l'insertion professionnelle de ces derniers au sein de l'entreprise. 
Devant ces défis, il est essentiel pour les managers de réfléchir à des moyens et des outils permettant une plus grande coopération entre des individus différents au sein de l'organisation. L'amélioration de la coopération passe ici par une meilleure capacité à communiquer (établir une relation entre ses différentes parties), un plus grand engagement de la part d'un nombre suffisant de membres (l'organisation doit ainsi être capable de capter puis de sécuriser la contribution de ses membres au niveau qui est nécessaire à l'atteinte de ses objectifs), et une volonté d'accomplir une finalité commune?. Quels moyens les gestionnaires pourraient-ils utiliser pour répondre à ces urgences?

\section{Un bref cadrage théorique : la notion de lieu}

Pour répondre à cette question, nous utilisons une approche fondée sur le lieu (place-based), car nous pensons que cette notion est au cœur des questions sur la nature des relations de travail entre les immigrants et les travailleurs locaux. En faisant une synthèse de la plupart des contributions sur la notion de lieu, nous retiendrons la pluridimensionnalité du concept, soulignée par Guthey et al. (2014) autour de trois caractères: 1) géographique; 2) local; 3) symbolique (« sense of place ») ${ }^{8}$. Le lieu est à la fois un emplacement géographique précis; dans ce sens, il est physique, tangible et concret, et fait partie de notre environnement naturel. Le lieu est également un cadre direct de l'expérience humaine, composé par les relations informelles et institutionnelles (d'ordres économique, politique, culturel, historique, communautaire, organisationnel, etc.) entre les acteurs sociaux appartenant à un même espace. Toutefois, le lieu est aussi le produit d'une expérience humaine vécue; il embrasse des dimensions culturelles et sociales qui sont assignées par les individus, les groupes, les communautés et les organismes qui y vivent et y travaillent. Il est composé d'interactions, d'expériences, de sens, d'émotions, et d'un paysage matériel où les humains se sentent chez eux dans leur monde et à leur place; « un endroit est un morceau de tout l'environnement qui a été revendiqué par des sentiments $»$. De la même manière que la notion de distance (perçue a priori sous l'angle essentiellement géographique) est mesurée sous les angles à la fois physique et interactionnel ${ }^{10}$, le lieu doit être abordé comme une construction conceptuelle aux multiples facettes.
D'autres auteurs ont distingué différentes conceptions du lieu au moyen de plusieurs principes définitionnels distincts ${ }^{11}$ : un principe d'emplacement qui suppose que le lieu renvoie à un positionnement physique précis et objectif; le lieu est ici une coordonnée objective sur la surface de la Terre; un principe d'épaisseur/consistance selon lequel le lieu ne peut être réduit en un point; il a une épaisseur physique et intangible, une consistance, une forme (gestalt), deux choses en un même lieu ne pouvant être fusionnées l'une l'autre; un principe de délimitation spatiale, selon lequel un lieu ne peut pas exister s'il ne peut être distingué des autres endroits. Toute définition du lieu doit donc préciser comment un lieu spécifique se distingue des autres; un principe d'unicité d'après lequel le lieu est unique; les lieux se distinguent les uns des autres du fait de la coexistence de la multiplicité des interprétations individuelles que nous en avons. Ce principe d'unicité est au cœur de l'appréhension du lieu comme une construction sociale qui tend à dominer la littérature scientifique actuelle. En tant que tels, les lieux sont intrinsèquement des constructions humaines. Le qualificatif « humain » ne se reporte pas à l'individu pris isolément. Il se réfère plutôt à l'individu socialisé.

Tous les individus appartenant à un groupe partagent notamment une compréhension sociale de ce qui fait l'unicité d'un lieu. Ầ ce titre, ce principe est au cœur de l'émergence de diverses interprétations concurrentes de la notion de lieu. Ainsi, au-delà des caractéristiques physiques remarquables, l'élément immatériel est largement considéré par les chercheurs comme un élément central dans l'établissement, la diffusion et le maintien de l'unicité perçue du lieu. Cette socialité est d'ailleurs source de renforcement des capacités de développement des communautés au sein d'un lieu; c'est ce que Bruno Jean appelle « capital social ». Selon ce dernier, l'ouverture à la diversité, la capacité de travailler ensemble, la qualité des liens entretenus et les réseaux tissés avec la communauté influencent le développement et la prospérité des communautés locales ${ }^{12}$. Le principe suivant est lié à l'interrelation/l'ouverture entre les lieux, ce qui suppose une interdépendance entre les lieux; ce principe renvoie aux influences potentielles d'un lieu sur un autre et le degré d'ouverture des lieux. Ensuite, le principe de temporalité suppose une vision dynamique du lieu, une constante évolution de ce dernier, et enfin le principe d'arrangement scalaire suppose une prise 
en compte de la taille du lieu et des différentes échelles qui s'y côtoient; le concept de lieu est souvent compris comme désignant quelque chose de très petite échelle; il est souvent confondu (à tort) avec l'échelle locale.

Au vu de ces principes définitionnels, on comprend qu'un lieu géographique est intrinsèquement dynamique et multiple, c'est-à-dire qu'il contient les diverses histoires, représentations, interprétations et pratiques de ceux qui le vivent. Suivant cette logique, il est alors possible d'appréhender la diversité des travailleurs en confrontant les discours des uns et des autres au sujet d'un lieu physique donné. D'où notre idée d'entreprendre une activité de promenade révélant leur diversité aux participants qui sont invités à partager leurs perceptions de divers lieux patrimoniaux de la ville de Montréal, à raconter leur vécu professionnel et à réfléchir à l'impact de ces différences sur leurs pratiques de coopération au travail dans l'entreprise.

\section{La « Promènade ${ }^{13}$ ", principes de base}

L'activité que nous avons intitulée «Promènade» trouve sa pertinence essentiellement dans la littérature. Déjà, Raulet-Croset (2011) explore comment la combinaison de l'approche qualitative de shadowing et l'approche ethnographique peut être utilisée pour analyser les dimensions spatiales et lieuitaires, où le lieu est appréhendé comme une coconstruction et une association des pratiques à la fois individuelles et collectives. L'auteur s'intéresse particulièrement aux méthodes d'investigation qui offrent une meilleure compréhension du rôle de l'espace dans les organisations, en particulier celles qui parviennent à saisir la dimension spatiale dans les pratiques quotidiennes d'une organisation. Elle soutient ainsi que les promenades commentées offrent des preuves empiriques et l'accès analytique à la dimension spatiale des pratiques, à partir de trois activités interreliées : marcher, parler et être avec $^{14}$. La promenade à pied est un dispositif écologique qui permet de faire évoluer les pensées, agissant en tant que « règle directrice»; c'est une activité qui libère l'esprit et permet aux pensées de vagabonder. Le fait de " marcher» est donc fondamental. Elle permet d'identifier certains «signes » qui peuvent rafraîchir la mémoire et provoquer des récits, et aussi de vivre une relation réelle avec l'espace.
Dans le même esprit, Mirabelle da Palma (2005) considère la marche comme une technique du corps partagée par le plus grand nombre et pouvant être employée pour appréhender le territoire autrement que par une grille fonctionnaliste; la promenade place dans ce sens l'individu en relation à luimême, à l'autre et à l'espace. Utilisée comme un outil, elle débouche sur la construction d'un savoir sur l'espace, ses usages, ses perceptions, ses vécus, et ses malentendus. Elle engendre un lien, une relation particulière avec le territoire et avec tous les acteurs qu'elle met à contribution, elle produit un savoir multiforme, qui pourrait se solder par un nouveau sens du lieu ${ }^{15}$.

Par ailleurs, Daskalaki (2007) ${ }^{16}$ traite de la façon dont la ville où est implantée une entreprise structure l'expérience de ses habitants; les activités d'un groupe de personnes au cours d'une promenade sont décrites et la promenade y est expliquée comme une métaphore, mais aussi comme une source d'inspiration pour discuter de la nécessité des structures spatiales de la ville et de leur influence sur le lieu de travail. L'auteur utilise ici la promenade de façon originale et engageante et suppose une profonde relation réciproque avec le paysage urbain. Il décrit la dialectique de la relation entre l'espace, le corps et l'environnement bâti. L'espace n'est pas vu simplement comme un élément distinct lié à la pratique organisationnelle, mais comme quelque chose qui revêt une histoire et un sens. La promenade dans ce sens est une activité par laquelle l'existence de l'espace est réaffirmée et partagée à tous les individus. L'objectif principal de l'article est ainsi de développer et d'étendre une nouvelle vision de l'espace; comment donner du sens aux «non-lieux » qui caractérisent l'espace urbain et les transformer en lieux de créativité et de dialectique. Il s'agit ici de percevoir l'espace plutôt comme un environnement construit par des expériences individuelles et partagées au cours de l'activité.

David Le Breton (2000) propose pour sa part un énoncé des vertus de la marche, présentée comme une manière propice de prendre de la distance à l'égard du rythme effréné de la vie quotidienne et d'affûter ses sens. Il réalise une interprétation sociologique de la marche dans la ville, en rappelant que ce comportement est une «mise en jeu constante » de l'expérience individuelle ${ }^{17}$. Pouvant prendre la forme d'une simple immersion dans la nature 
frayant un chemin vers soi et vers les autres, d'un moyen de se rassembler et de s'ouvrir au monde ou encore d'un profond désir pour des horizons inconnus, la marche constitue une forme de résistance à l'hypermodernité, tout en permettant une meilleure connaissance de soi, des autres, des lieux ou des choses.

Ces écrits mis ensemble nous permettent de justifier une approche par la marche et la promenade, pour mieux appréhender le lieu et les perceptions individuelles suscitées par le passage au sein de différents lieux. Cette approche permet de comprendre le sens du lieu des individus et le faire partager aux autres. Notre objectif ici est de produire un savoir multiforme sur la base de la contribution de chacun des participants; saisir ce que représente un lieu spécifique pour chacun des individus et le faire partager aux autres participants pourrait non seulement susciter un plus grand intérêt pour le territoire et donc un désir de mieux le découvrir et le développer, mais aussi être source d'amélioration de la coopération organisationnelle dans la mesure où une compréhension du lieu et de sa signification pour les individus à l'extérieur des organisations pourrait aider à mieux cerner les problèmes à l'intérieur de l'organisation.

\section{Description de l'activité et éléments clés}

L'activité consiste à concevoir et parcourir avec des travailleurs un itinéraire en marchant au sein de la zone géographique d'implantation de leur entreprise, dans notre cas, la ville de Montréal. Sa particularité réside dans l'itinéraire à suivre qui est constitué d'un lieu choisi par chacun des participants et inconnu des autres. En effet, chacun des participants à l'activité est chargé d'indiquer deux lieux géographiques patrimoniaux qu'il souhaite faire découvrir aux autres; en fonction des contraintes de temps ou de climat, de la proximité géographique entre les lieux choisis, mais surtout de leur caractère patrimonial (c'est-à-dire qu'ils relèvent a priori d'une connaissance commune minimale et d'un accès relativement public). Les animateurs (chercheurs dans notre cas) de la marche choisissent un des deux lieux proposés. Très souvent, le participant qui propose ses deux idées de lieu n'est pas lui-même informé du lieu qui sera finalement choisi par l'encadreur et intégré dans l'itinéraire. L'itinéraire final à suivre n’est dévoilé qu'au début de l'activité. L'idée sous- jacente dans ce choix est de ne susciter aucune préparation préalable de la part des participants qui doivent seulement se présenter tels quels, avec leurs connaissances et leurs émotions pour le lieu à partager. Une précision est néanmoins apportée quant à la ville dans laquelle va se dérouler l'activité. Le principe de la «Promènade » consiste ainsi en une visite guidée animée par et pour les participants à l'activité, passionnés par un lieu particulier qu'ils désirent présenter aux autres dans le périmètre géographique du lieu de leur activité professionnelle.

L'objectif principal d'une telle activité est de partager avec d'autres individus une perception individuelle d'un lieu patrimonial qui revêt une histoire publique et connue, mais dont les interprétations et les émotions qu'il suscite sont susceptibles de varier d'une personne à une autre, ceci en raison de ses origines, de ses valeurs ou de ses croyances. Il s'agit dès lors de côtoyer au travers de cette activité des émotions personnelles, plus profondes et existentielles sur un lieu dont l'histoire est pourtant connue et partagée par tous. En effet, cette sorte d'introspection permettrait finalement de mieux se découvrir soi-même, mais aussi de mieux découvrir les autres par des rapports plus authentiques. L'idée ici est donc de créer le dialogue par le rapprochement d'individus entre eux en utilisant un lieu particulier et signifiant; elle permet de faire naître une curiosité pour un lieu, de souder les individus et de créer un sentiment d'appartenance à une communauté ou à un lieu particulier. De plus, elle focalise l'attention sur les perceptions, les centres d'intérêt et les observations des individus pour un lieu patrimonial précis et permet aux participants de partager leurs observations et leurs souvenirs dans ce lieu (le cas échéant).

Selon l'objectif poursuivi, les participants à l'activité peuvent soit faire partie de la même entreprise, soit se situer dans des tranches d'âge relativement similaires, soit partager ou avoir partagé une même expérience lors de leur arrivée au Québec, ou évoluer dans le même secteur d'activité, etc. Il n'existe pas de critère particulier qui prédomine sur un autre. Par ailleurs, une parité en genre serait fort appréciable. L’idée est de réfléchir sur la différence, et indirectement sur les facteurs de succès de la coopération et de l'insertion organisationnelle, à partir d'une discussion sur le lieu.

Quant aux qualités personnelles nécessaires pour les animateurs comme pour les participants, il faut 
noter que la « Promènade » est plus une conversation en mouvement qu'un monologue de l'un des participants. Ces derniers doivent ainsi être généreux, désireux de partager leurs expériences, leur savoir et leurs croyances avec d'autres personnes. Ils doivent également avoir un bon sens de l'écoute, teinté de spontanéité pour intervenir constructivement dans les propos des autres, sans toutefois porter de jugement de valeur sur les propos, quels qu'ils soient. Si tel n'est pas le cas, il est du rôle de l'animateur de la promenade de s'assurer de la qualité du dialogue.

En ce qui concerne le nombre de participants souhaitable, il varie en fonction du temps disponible pour réaliser l'activité et du nombre d'animateurs. De plus, nous avons constaté que dans une limite de deux heures, trois arrêts (trois lieux) sont largement suffisants afin de garder l'attention de tout le groupe pendant toute la durée de la promenade.

Quant aux instructions liées au déroulement de l'activité proprement dite, elles consistent essentiellement dans l'écoute de la présentation de l'un des participants qui explique un lieu patrimonial de Montréal qui lui tient à cœur; il convient de préciser qu'il n'existe pas de bonne ou de mauvaise présentation et qu'aucune préparation préalable ne s'avère nécessaire; la possibilité est donnée aux participants de poser des questions lorsqu'ils le désirent; toutefois, la moquerie n'est pas permise. Quelques questions de départ peuvent néanmoins aider à planifier la marche, et à stimuler le débat pour en tirer les informations désirées.

\section{Un cas concret, résultats et contributions}

Afin de tester la fécondité de ce dispositif, nous l'avons mis en œuvre avec trois employés immigrants, appartenant à différentes entreprises. Grâce à cette promenade guidée/guidante par/pour nos interlocuteurs, nous espérions accéder au «sense-ofplace » de chacun dans une découverte collective et mutuelle, contribuant peut-être à un meilleur attachement, un enracinement des employés étrangers au sein de leur lieu d'accueil.

Nous avons ainsi planifié l'activité de promenade en fonction de la disponibilité des participants. Trois lieux furent identifiés : l'École polytechnique de Montréal, l'Oratoire Saint-Joseph du MontRoyal et la plus ancienne succursale de la librairie
Renaud-Bray sur le Chemin de la Côte-des-Neiges. L'activité s'est déroulée en deux temps: marche commentée (2 heures) puis débriefing en groupe de discussion (30 minutes). Toute l'activité fut enregistrée numériquement et l'essentiel des interactions a été ensuite retranscrit sous forme de comptes rendus exhaustifs. Il ressort de l'analyse de ceux-ci trois principaux éléments :

\section{Des perceptions du lieu différentes pour chacun des participants}

Si les perceptions des lieux géographiques sont pour la plupart similaires, les perceptions des lieux sur les plans local et «sense of place » sont quant à elles assez particulières et dépendantes du parcours et de l'expérience de chacun des participants. Sur le plan architectural, un consensus se dégage généralement pour relever la «grandeur » des constructions et la « rigueur» du climat. Toutefois, en considérant le lieu sous l'angle du local ou celui du «sense of place», nous remarquons des différences de perceptions.

En effet, sur le plan local, certains sont essentiellement orientés vers leur réseau privé et personnel, leur réseau amical, avec lequel ils découvrent le caractère festif et la vitalité de Montréal, tandis que d'autres sont plus portés vers leur réseau professionnel (partenaires d'affaires et collègues de travail) ou scolaire, qui les aidera dans la réalisation de leurs objectifs professionnels.

S'agissant du « sense of place», certains soulignent le caractère festif et la diversité de la ville comme des éléments traduisant un potentiel d'affaires important de la ville. Le lieu a ainsi une fonction utilitariste dans la mesure où il permet d'avoir accès à des contacts importants et essentiels pour l'atteinte des objectifs professionnels ou personnels. D'autres se penchent sur l'attractivité et la forte influence de la culture nord-américaine; une certaine attraction pour le « rêve américain » qu'ils espèrent réaliser en s'installant dans ce lieu. Le lieu revêt ainsi un sens plus émotionnel dans le sens où les travailleurs recherchent ici une relation plus personnelle avec leurs collègues de travail par l'organisation d'activités extraprofessionnelles, ou par l'agrandissement de leur réseau amical et personnel qui leur permettra de mieux découvrir et de mieux s'imprégner ledit lieu. Dans ce sens, la coopération s'avère intraprofessionnelle avec les collègues de bureau avec qui on essaie parfois, assez superficiellement 
quand même, de découvrir cette vitalité et ce caractère festif du lieu, mais surtout extraprofessionnelle, avec les amis et connaissances qui nous accompagnent au quotidien.

Pour d'autres employés en revanche, ils expriment un fort attachement à leur lieu de travail, qu'ils qualifient de convivial et au sein duquel règnent entraide et partage. Par contre, ils restent focalisés sur un retour inconditionnel à leurs origines; ils vouent un attrait irrésistible à leur pays d'origine; ils désirent y réaliser des investissements importants, malgré le degré d'attractivité élevé de ces derniers pour leur lieu de travail ou pour la ville de Montréal; le lieu représente ainsi une sorte de transition nécessaire pour atteindre cet objectif; ils sont ainsi soit à la recherche de meilleurs partenaires professionnels locaux ou étrangers pour les aider dans ce projet, soit, ils essaient de s'intégrer au maximum dans leur lieu de vie et surtout de travail, grâce à un enracinement dans la culture du lieu, afin de garder un maximum de contacts professionnels, de partage d'expériences et d'expertises qu'ils pourront mettre à profit au sein de leur pays d'origine afin de réaliser leurs projets de vie. Le lieu est perçu comme une sorte de transition, une étape nécessaire vers des aspirations futures.

Cette analyse nous permet ainsi de faire ressortir trois principales formes de "lieuité » (la qualité d'être un lieu) vécues par les travailleurs interrogés : il s'agit d'une « lieuité utilitariste et fonctionnelle » qui voit le lieu et les relations qu'on y entretient comme des moyens permettant d'atteindre un but prédéterminé et fixe; une « lieuité existentiellement urbaine » ou « émotionnelle » dans laquelle le lieu est cet endroit festif, vivant et culturellement attractif, dans lequel le partage et l'échange des cultures et des émotions sont encouragés, et enfin une «lieuité professionnelle transitoire » pour laquelle le lieu est cet espace de rencontres et d'échanges constructifs avec des professionnels de divers domaines, permettant à terme de se développer sur le plan professionnel. Un tableau récapitulant ces différentes formes de lieuités est présenté en annexe.

\section{Quelques principes définitionnels du lieu de Montréal}

Le tableau en annexe nous permet de définir Montréal comme elle a été appréhendée par les différents participants lors de la promenade à travers les différents lieux choisis. Pour ce faire, nous avons utilisé la grille d'analyse en sept principes constitutifs de la notion de lieu (localisation, consistance, délimitation, unicité, interrelation, temporalité, arrangement scalaire), telle qu'élaborée par Sergot et Saives (2016) dans leur revue de la littérature sur ladite notion.

Le principe de localisation renvoie à la situation de Montréal et à l'emplacement géographique des lieux spécifiques choisis dans le monde. En effet, Montréal est une ville située géographiquement en Amérique du Nord, mais, à travers les espaces visités (une école d'ingénieurs, un lieu religieux et une librairie), elle représente également un lieu de connaissances dans lequel l'acquisition des savoirs occupe une place primordiale; un lieu d'expérimentation avec l'accent donné à la pratique et à l'expérimentation, un lieu de culte dans lequel la religion catholique occupe une place centrale, un lieu d'apprentissages multidisciplinaires où la polyvalence dans les disciplines est de plus en plus prisée, un lieu de partage et de différences dans lequel chaque individu apporte sa particularité et ses différences et les partage avec les autres.

Le principe d'épaisseur ou de consistance suppose que le lieu ne peut être réduit en un point; il a une épaisseur à la fois matérielle et intangible. Dans notre cas, les lieux visités nous révèlent que Montréal est une ville de tous les possibles : la grandeur a une place symbolique et s'observe dans la dimension des bâtiments et des constructions, mais aussi dans l'envergure que prennent les projets, et même dans les rêves des individus; une sorte d'American Dream a été constaté dans les entrevues et les discours de la plupart des participants, une grandeur qui fait dire à l'un de nos participants qu' «il ne faut pas voir les choses en petit ». Montréal se caractérise également par l'accès qu'elle donne au savoir scientifique et pratique, à la culture générale, à la mobilité et à l'ouverture internationale. Toutefois, la ville est aussi un lieu de malaises, dans lequel le coût des études, le culte de la performance (économique comme scolaire), le caractère tabou de la spiritualité et de la pratique religieuse et la prédominance du superficiel sur le spirituel constituent très souvent les réalités sombres de cette consistance hétérogène et plurale.

Le principe de délimitation spatiale suppose de définir en quoi et comment un lieu peut être porteur de 
limites ou de frontières. Dans le cas particulier de Montréal, les participants nous ont fait part de l'existence de frontières parfois culturelles ou raciales quant à l'évolution au sein de l'entreprise; il existerait ainsi une limite dans la hiérarchie au sein de certaines entreprises pour les travailleurs immigrants. Par ailleurs, on observe plutôt une absence de frontières dans la délimitation entre la vie privée et la vie professionnelle; l'accent au sein des entreprises est mis sur la réalisation des objectifs, au fait de «faire son travail » plutôt qu'à la présence en entreprise, au fait d'«être au travail». Montréal suppose en outre cette idée de volume, d'espace, une symbolique de l'amplitude qui nous fait penser que l'horizon spatial est immense et étendu.

Le principe d'unicité suppose que le lieu est unique, mais non unitaire; chaque lieu se distingue des autres du fait de la coexistence de la multiplicité des interprétations individuelles que nous en avons. Ce principe est d'ailleurs ce qui est ressorti principalement de nos travaux. En effet, chacun des participants a sa propre vision de Montréal, de ses éléments géographiques, de ses spécificités locales ou culturelles et chacun y est sensible d'une manière différente. Les entrevues et la promenade nous ont permis de mieux comprendre cette réalité; ce qu'il en ressort nous permet de dire que Montréal est unique du fait de son climat, perçu tantôt comme invivable, tantôt comme gérable; ensuite, l'unicité de Montréal s'observe par sa diversité culturelle, ses festivités en tout genre, perçues tantôt comme un potentiel d'affaires, tantôt comme un moyen d'apprentissage ou de découverte de l'autre. Montréal est également unique du fait du bon compromis qu'elle propose entre la métropole et le village (paysage, ruralité, espaces verts). Comme nous l'avons dit précédemment, la ville est également celle où les mots et expressions tels que grandeur, espace, accès à la connaissance pratique, culture générale trouvent tout leur sens, mais encore une fois, restent teintés par les parcours particuliers des individus qui les interprètent chacun à leur manière.

Le principe d'ouverture ou d'interrelation fait référence à l'interdépendance entre les lieux, une influence potentielle d'un lieu sur un autre. Là encore, les discours des participants lors de la promenade nous présentent Montréal comme un lieu ouvert et plus ou moins influencé par d'autres lieux; en effet, la construction du discours sur le lieu s'opérait chaque fois dans la comparaison à d'autres lieux; les participants mentionnaient systématiquement leurs expériences en France ou au Cameroun pour les comparer à leur vécu à Montréal; cette comparaison s'est ainsi observée dans l'apprentissage (apprentissage théorique en France versus apprentissage pratique à Montréal), dans le style des constructions et des routes (routes assez petites en France versus routes immenses à Montréal), dans les célébrations religieuses (célébrations très conviviales et collectives au Cameroun versus célébrations plus courtes et personnelles à Montréal), dans le travail (bureaucratie excessive en France versus accent mis sur les objectifs à Montréal), etc. De plus, la situation de la ville en Amérique du Nord a été relevée comme un des facteurs explicatifs de la culture et de la conception américaine qui y est observée (caractère pratique et pragmatique de l'apprentissage). Par ailleurs, l'influence de l'histoire franco-amérindienne sur la culture montréalaise a également été mentionnée. En outre, à plusieurs niveaux, Montréal apparait comme une ville mixte et diversifiée sur le plan culturel, caractérisée par une multiplicité de cultures diverses.

En matière de temporalité, Montréal véhicule une dynamique de court ou de moyen terme, en même temps qu'une stabilité de l'instant présent. Les individus s'envisagent à partir de leur expérience présente à Montréal. Dans un horizon qui reste celui du court terme, en quelque sorte, Montréal est une ville à temporalité courte ou accélérée où les projets et le rythme des projets sont ceux du court terme : la durée d'un emploi à un poste est souvent de deux ans, et même le monde scolaire est aux prises avec cette temporalité de l'accélération (la course à la publication et à la performance à court terme). Par ailleurs, la perspective d'un départ de Montréal mentionné par la plupart des travailleurs nous permet d'envisager une temporalité projective de moyen ou de long terme. La temporalité est également celle du présent avec certains récits sur les expériences extraordinaires qui sont en cours dans le lieu.

Enfin, le principe d'arrangement scalaire renvoie à la taille du lieu et à la façon dont différentes échelles s'y côtoient. Dans ce sens, Montréal est porteuse d'arrangements scalaires variés; on y retrouve un monde à la fois urbain, caractéristique des grandes métropoles, mais aussi un monde rural et de banlieue. De plus, les aspirations quittent la sphère du 
local pour s'envisager davantage à l'échelle internationale. Enfin, une aspiration à un retour à la vie du village s'observe chez certains participants pourtant essentiellement urbains.

Après cette présentation du lieu dans le cas étudié, nous pouvons affirmer avec Sergot et Saives (2016) que le lieu n'est pas une construction unitaire ni homogène. Il est plutôt une construction dynamique, aux multiples facettes. Nous faisons en outre l'hypothèse que l'activité de promenade des travailleurs révèle et affecte leurs relations de travail au sein de l'entreprise. Ce que nous essayerons de déceler dans la prochaine partie.

\section{Une redéfinition de la relation d'emploi : coconstruction des perceptions individuelles du lieu pour une meilleure coopération organisationnelle}

De ce qui précède, nous pouvons établir plusieurs relations entre la lieuité (la qualité d'être un lieu) et la coopération organisationnelle. En effet, la plupart des discours et des interventions font état de l'influence des représentations et expériences du lieu sur la relation d'emploi et le travail; partant de l'expérience d'un site patrimonial religieux, un des participants évoque la notion d'éthique pour signifier le fait que sa culture religieuse lui impose une sensibilité particulière à l'adoption de comportements éthiques au travail. De plus, il fait un rapprochement entre l'impression de grandeur ou de volume ressentie dans l'expérience de l'architecture urbaine métropolitaine et le travail en entreprise. En effet, ces termes clés caractérisent également le travail au sein de son entreprise et l'envergure des projets confiés. Devant l'étalage d'ouvrages en librairie, un autre participant nous parle de la nécessaire culture générale dans son travail quotidien afin d'être porteur d'innovation. En effet, la culture et les idées qu'il acquiert dans les livres lui donnent les ressources nécessaires pour performer et innover au quotidien au sein de l'entreprise, et même plus tard, dans son projet d'entrepreneur. Le lieu apparait ainsi comme une source d'inspiration pour des idées créatives, une source d'inspiration pour une nouvelle manière de gérer, différente des conceptions apprises jusque-là. Ainsi, la culture générale traduit cette multidisciplinarité qui est de plus en plus encouragée et cet excès de spécialisation (hyperspécialisation) de plus en plus critiqué. Dans le même sens, le troisième participant fait le lien entre la grandeur des espaces de Montréal et la possibilité de nourrir son imaginaire, porteur d'idées innovantes dans son travail quotidien.

De ce qui précède, nous déduisons que l'amélioration de la coopération organisationnelle passerait par:

- Une prise en compte et un partage des « sense of place » individuels afin de profiter de la complémentarité des expériences et des savoirs individuels; cela suppose un cadre de délibération de ces perceptions individuelles afin d'aller au-delà du simple rapport de travail souvent factice et de côtoyer des éléments plus personnels et identitaires, permettant de comprendre le comportement et la façon de travailler des collègues et des employés. En effet, une telle activité est en soi porteuse d'une nouvelle socialité, moins superficielle et plus authentique, mais surtout une socialité qui s'installe dans le temps, et qui influencerait positivement le travail au sein de l'entreprise. Cette dernière transparait dans le désir formulé par les participants de poursuivre l'expérience et de faire partager avec leurs collègues de bureau les différents lieux découverts durant l'activité de promenade. Les interventions dans ce cadre nous montrent ainsi des rapports de collaboration qui sont pour le moment assez superficiels et limités au strict cadre professionnel; les participants aimeraient ainsi faire partager leurs découvertes, afin de sortir un peu du moule des conversations convenues au travail, partager ce qu'ils sont et en apprendre davantage des autres.

- Une prise en compte du lieu/de la lieuité comme une source d'inspiration, de créativité, de vitalité, d'ouverture pour les organisations; une telle activité stimule l'imaginaire et contribue à encourager la créativité chez les employés; - Une prise en considération des différentes dimensions du lieu et de la façon dont chacune d'elles est appréhendée par les employés durant la prise de décisions organisationnelles, notamment en matière d'attribution des postes ou de constitution d'équipes de travail hétérogènes.

On comprend dès lors que le lieu et les perceptions individuelles du lieu influencent à divers niveaux les comportements individuels au travail, la coopération entre collègues et même le rendement. Spécifiquement, les éléments saillants tels que l'éthique, 
l’idée de grandeur, la connaissance, la culture générale, et même l'idée d'authenticité et de socialité recensées à partir des lieux visités, trouvent écho au sein des organisations et influenceraient le travail quotidien.

\section{Conclusion}

Les moyens d'ajustement des méthodes de gestion mises en place au sein des entreprises dans une perspective régulatrice n'ont pas manqué depuis l'injonction faite au management de prendre en compte la diversité culturelle. Si de tels moyens ont donné lieu à un encadrement mieux défini et plus circonscrit pour que s'engagent les changements souhaités, d'autres seraient encore souhaités et espérés pour que s'opèrent de l'intérieur les modifications au sein des groupes de travail. Dans ce sens, l'initiative proposée ici de mettre de l'avant les concepts de lieu et de lieuité et d'étudier les perceptions qu'ont les individus et les employés de leur lieu géographique d'implantation se veut de ceuxlà. Ainsi, l'analyse des discours recueillis au cours de notre promenade nous a fourni un éclairage sur le lien entre lieuité et coopération organisationnelle, en révélant d'abord trois formes de « lieuité » caractérisant les employés interrogés, en validant ensuite une approche multidimensionnelle et évolutive du concept de lieu, et en identifiant enfin une série d'éléments « lieuitaires » (l'éthique, l'idée de grandeur ou d'envergure, la connaissance scientifique et pratique, la culture générale ou l'ouverture multidisciplinaire et surtout une socialité authentique et installée dans le temps) porteurs d'intégration et de socialisation organisationnelle. De plus, l'activité de promenade constitue en soi un nouvel outil de gestion pouvant être réutilisé pour cerner les « sense of place » individuels, les mettre en commun et les partager au sein d'équipes de travail, pour une meilleure collaboration au sein de l'entreprise. Sans avoir la prétention de généraliser nos résultats, nous avons voulu offrir ici un cadre permettant à tout individu désirant répéter l'activité de « Promènade» de le faire. Nous pensons qu'elle pourrait parfaitement s'intégrer dans les activités de Team building souvent légion au sein des entreprises multiculturelles.

\section{NOTES}

1 Ici Radio-Canada (2016). Nombre record d'immigrants admis au Canada. Repéré à http://ici.radio-canada.ca/ nouvelle/805803/immigrants-record-refugies-syriens-statistique-canada

2 Zietsma, (2010). Immigrants exerçant des professions réglementées. Statistique Canada, 15-31. Repéré à http://www.statcan.gc.ca/pub/75-001-x/2010102/article/11121-eng.htm

3 Ici Radio-Canada (2013). Le Canada devient de plus en plus un pays d'immigrants. Repéré à http://ici.radiocanada.ca/nouvelles/National/2013/05/08/001-statistique-canada-immigrants-minorites-visibles.shtml

4 Ici Radio-Canada (2015). Quelque 50000 immigrants attendus au Québec en 2016. Repéré à http://ici.radiocanada.ca/nouvelle/746999/quebec-immigration-cibles-2016-refugies-syriens

5 Ici Radio-Canada (2013). Le Canada devient de plus en plus un pays d'immigrants. Repéré à http://ici.radiocanada.ca/nouvelles/National/2013/05/08/001-statistique-canada-immigrants-minorites-visibles.shtml

6 Arcand, S. (2006). Gestion des opérations, de la logistique, de la production et des services : prévenir les conflits liés à la diversité. Revue de Gestion, HEC Montréal, 31(4), 216-223.

7 Barnard, C. I. (1938). The functions of the executive. Cambridge, MA: Harvard University Press.

8 Guthey, G. T., Whiteman, G. et Elmes, M. (2014). Place and sense of place: Implications for organizational studies of sustainability. Journal of Management Inquiry, 23(3), 254-265.

9 Shrivastava, P. et Kennelly, J. J. (2013). Sustainability and place-based enterprise. Organization \& Environment, 26(1), 83-101.

10 Charron, M. et Shearmur, R. (2005). Distances, interactions et analyse spatiale de la ville: le cas de Montréal. Revue d'économie régionale et urbaine, (2), 163-192.

11 Sergot, B. et Saives, A. L. (2016, juin). Placing organized work: how and why place is a useful concept for Management and Organization Studies. Communication présentée à la Conférence de l'EURAM.

12 Jean, B. et Wa, A. É. M. (2004). «Le capital social et le renforcement des capacités de développement des communautés rurales: les enseignements d'une étude canadienne. Revue d'économie régionale et urbaine, (5), 673-694. 
13 Nous avons intitulé l'activité « Promènade » pour marquer le fait que la marche est menée, c'est-à-dire conçue et guidée par les choix des animateurs à partir des propositions des participants.

14 Raulet-Croset, N. et Borzeix, A. (2014). Researching spatial practices through commentated walks:“on the move" and "walking with". Journal of Organizational Ethnography, 3(1), 27-42.

15 Mirabelle, Da Palma, E. Brito (2005, octobre). La promenade, un outil de connaissance du territoire. Communication présentée à la troisième Conférence de l'intelligence territoriale. Territory, wellbeing and social inclusion, REIT. Liège, Belgique.

16 Daskalaki, M., Stara, A., et Imas, M. (2008). The 'Parkour Organisation': inhabitation of corporate spaces. Culture and Organization, 14(1), 49-64.

17 Le Breton, D. (2000). Chemin de traverse : Éloge de la marche. Métailié, 44(1), 5-16. 


\section{ANNEXE : les différentes formes de « lieuités »}

\begin{tabular}{|c|c|c|c|}
\hline & $\begin{array}{l}\text { Lieuité utilitariste } \\
\text { et fonctionnelle }\end{array}$ & $\begin{array}{c}\text { Lieuité existentielle } \\
\text { urbaine }\end{array}$ & $\begin{array}{c}\text { Lieuité professionnelle } \\
\text { transitoire }\end{array}$ \\
\hline $\begin{array}{l}\text { Dimension physique } \\
\text { ou géographique }\end{array}$ & $\begin{array}{c}\text { Situation géographique } \\
\text { de la région et proximité } \\
\text { des USA: } \\
\text { Possibilité d'être rapide- } \\
\text { ment bilingue en s'y } \\
\text { installant }\end{array}$ & $\begin{array}{l}\text { Situation en Amérique du } \\
\text { Nord: sorte de rêve } \\
\text { américain où l'on a } \\
\text { l'impression que tout est } \\
\text { possible }\end{array}$ & Proximité avec les USA \\
\hline Dimension locale & $\begin{array}{l}\text { Montréal } \\
\text { représente un lieu } \\
\text { de contacts et de } \\
\text { potentiel d'affaires } \\
\text { important } \\
\\
\text { Potentielle présence de } \\
\text { partenaires d'affaires } \\
\text { dans la région; un réseau } \\
\text { professionnel immense; } \\
\text { importance des activités } \\
\text { de réseautage pour la } \\
\text { croissance de l'entreprise } \\
\text { Relation interpersonnelle } \\
\text { avec le client ou le } \\
\text { partenaire d'affaires }\end{array}$ & $\begin{array}{l}\text { Montréal représente } \\
\text { un lieu urbain, festif et } \\
\text { à forte mixité sociale } \\
\text { Lieu de travail : socialité } \\
\text { assez superficielle (répartition } \\
\text { des employés par bureau, } \\
\text { création de groupes informels) } \\
\text { Lieu de vie : Présence } \\
\text { d'un réseau essentiellement } \\
\text { amical et communautaire (la } \\
\text { socialisation est surtout } \\
\text { produite sur le plan personnel } \\
\text { avec des individus de la même } \\
\text { communauté) } \\
\text { Forte possibilité d'agrandir ce } \\
\text { réseau }\end{array}$ & $\begin{array}{l}\text { Montréal représente un } \\
\text { lieu attractif et inspirant, } \\
\text { mais retour inéluctable } \\
\text { vers le pays ou le } \\
\text { continent d'origine } \\
\text { Lieu de tra vail : } \\
\text { Opportunités de faire grandir } \\
\text { son réseau professionnel: } \\
\text { activités de réseautage et } \\
\text { réseaux sociaux numériques } \\
\text { Relations professionnelles } \\
\text { superficielles, mais basées sur la } \\
\text { convivialité, l'entraide et le } \\
\text { partage } \\
\text { Lieu de vie: } \\
\text { Réseau amical essentiellement } \\
\text { étranger (issu du pays d'origine) } \\
\text { Possibilité d'implication dans } \\
\text { la communauté québécoise à } \\
\text { travers une activité sportive } \\
\text { Possibilité d'intégrer des asso- } \\
\text { ciations culturelles pour une } \\
\text { meilleure connaissance des } \\
\text { cultures : culture du groupe } \\
\text { plutôt que de l'individu }\end{array}$ \\
\hline $\begin{array}{c}\text { Dimension } \\
\text { " sense of place " }\end{array}$ & $\begin{array}{l}\text { Potentiel festif de la ville: } \\
\text { Présence dans le lieu } \\
\text { d'importants événements } \\
\text { festifs et attractifs, } \\
\text { traduisant un potentiel } \\
\text { d'affaires énorme pour le } \\
\text { développement de } \\
\text { l'entreprise }\end{array}$ & $\begin{array}{l}\text { Lieu de vie : Présence } \\
\text { d'activités festives et attractives } \\
\text { Potentiel touristique de la } \\
\text { ville } \\
\text { Ouverture des mentalités } \\
\text { Configuration urbaine du } \\
\text { lieu } \\
\text { Possibilité de rencontrer } \\
\text { davantage de monde } \\
\text { Lieu de tra vail : absence } \\
\text { de vitalité au sein de ce lieu } \\
\end{array}$ & $\begin{array}{l}\text { Lieu de vie : Diversité } \\
\text { culturelle, festivités, ambiance et } \\
\text { affluence dans certains } \\
\text { lieux publics (les marchés), } \\
\text { potentiel touristique, découvertes } \\
\text { bistoriques } \\
\text { Lieu de travail: } \\
\text { Convivialité, entraide et partage } \\
\text { d'expériences }\end{array}$ \\
\hline
\end{tabular}

